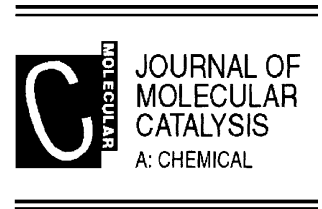

ELSEVIER

Journal of Molecular Catalysis A: Chemical 185 (2002) 211-222

www.elsevier.com/locate/molcata

\title{
Hydrogenation of naphthalene and methylnaphthalene: modeling and spectroscopy
}

\author{
Ş. Sayan ${ }^{\mathrm{a}, 1}, \mathrm{~J}$. Paul $^{\mathrm{b}, *}$ \\ a Department of Chemistry, Bilkent University, 06533 Bilkent-Ankara, Turkey \\ ${ }^{\mathrm{b}}$ Department of Chemistry, Hacettepe University, 06532 Beytepe-Ankara, Turkey
}

Received 21 June 2001; accepted 15 January 2002

\begin{abstract}
In situ infrared spectra of 1-methylnaphthalene (1-MeNapht) hydrogenation, over sulfided $\mathrm{NiMo} / \mathrm{Al}_{2} \mathrm{O}_{3}-\mathrm{TiO}_{2}$ catalysts, were compared with theoretically derived properties of methylnaphthalene and its bicyclic products: MeDilin, MeTetralin, MeOctalin and MeDecalin, and with conversion data from literature. Comparisons were also made between the un-substituted and methyl-substituted two-rings, and between the 1- and 5-methyl isomers of 1,4-dihydronaphthalene (dilin) and 1,2,3,4tetrahydronaphthalene (tetralin). IR spectra of MeNapht adsorption, on the sulfided catalyst, were matched with data for adsorption on the catalyst without sulfidation and the empty support.

Surface bound MeNapht is observed below $250^{\circ} \mathrm{C}$ on all catalysts. MeNapht adsorption suppresses $\mathrm{OH}$ groups nondiscriminatory on the empty support and the metal loaded catalyst. We relate the results to previous data on the interaction between the supported metal sulfides and titanium modified aluminas.

Calculated total energies, and experimentally derived heats of formation, pointed at decahydronaphthalene (decalin) as the dominant product of naphthalene hydrogenation, with tetralin as an abundant intermediate, and dilin and 1,2,3,4,5,6,7,8octahydronaphthalene (octalin) as short lived transient stages. The spectroscopic modeling showed that the orbital fingerprints of the five bicyclic compounds were not distinctly different, nor more than marginally modified by methyl substitution or isomerization. The only significant difference came at the highest occupied orbital, where a high naphthalene density of states (DoS) overlapped with the valence bands of metal or metal sulfide catalysts.

The vibrational bands for naphthalene, dilin, tetralin and octalin were well separated. Octalin and decalin, alone, have similar vibrational spectra. Upheaval of ring degeneracy for methyl-substituted two-ring structures broadened all infrared bands in a characteristic way. @ 2002 Elsevier Science B.V. All rights reserved.
\end{abstract}

Keywords: Methylnaphthalene; Naphthalene; Hydrotreatment; Modeling

\section{Introduction}

Hydroaromatization (HDA) or hydrogenation of polyaromatic compounds is a critical part of refinery operations, aiming at the conversion of heavy feeds to fuels [1-4]. Coal derived liquids, a viable and potentially very abundant feedstock to refineries, are similar to heavy fractions of crude. Their composition varies with the liquefaction process, but two-ring aromatics

\footnotetext{
* Corresponding author. Present address: Divison of Physics, Luleå University of Technology, 97187 Luleå, Sweden.

Tel.: +46-920-491793; fax: +46-920-491074.

E-mail address: jan.paul@mt.luth.se (J. Paul).

${ }^{1}$ Present address: Department of Chemistry, Rutgers University, Piscataway, NJ 08854, USA. Piscataway, NJ 08854, USA.
} 
are always abundant. Complete hydrogenation (HYD), followed by cracking under hydrogen, constitutes an attractive two-step process to convert these feeds to high quality gasoline or diesel [5-7]. The first step can be accomplished over sulfided metal catalysts and the second over $\mathrm{Pd} / \mathrm{zeolite}-\mathrm{X}$. Sulfidation lowers the dispersion of a supported metal phase, but metal sulfide particles gain the necessary hydrogen activity for hydrogenation at the appropriate reaction temperature. Metal catalysts, inherently with superior activity, cannot maintain a high hydrogen coverage at elevated temperatures, due to recombinative desorption.

Naphthalene (Napht) and methylnaphthalene (MeNapht) are model compounds for these feeds. Their low $\mathrm{H}: \mathrm{C}$ ratios (8:10 or 10:11) are increased by hydrogenation to dilin/MeDilin (10:10 or 12:11), tetralin/ MeTetralin (12:10 or 14:11), octalin/MeOctalin (16:10 or $18: 11)$ and decalin/MeDecalin $(18: 10$ or 20:11). Conversion and kinetics studies indicated that 1,2,3,4-tetrahydronaphthalene (tetralin) and decahydronaphthalene (decalin) are the more stable products, with 1,4-dihydronaphthalene (dilin) and $1,2,3,4,5,6,7,8$-octahydronaphthalene (octalin) as less abundant intermediates [6,8]. Successful cracking over zeolite-X catalysts must act on decalin, because it is only the completely hydrogenated two-ring which can pass readily through the pores of the support [9].

Thermodynamic properties were shown to provide a good estimation for product selectivity, thereby reducing the number of screening tests, with respect to reaction temperature [7]. Equilibrium constants for the conversion of aromatics to isoparaffins and cycloparaffins were calculated, to determine reaction conditions for the production of high octane gasoline components, without aromatics, from hydrogenation of 1-MeNapht, followed by hydrocracking of the fully hydrogenated product [10]. These calculations, combined with screening studies of different catalysts, determined optimum conditions $\left(325^{\circ} \mathrm{C}, 70 \mathrm{bar}\right)$ for complete hydrogenation of 1-MeNapht, using a presulfided $\mathrm{NiMo} / \mathrm{TiO}_{2}-\mathrm{Al}_{2} \mathrm{O}_{3}$ catalyst [8].

The role of chemisorption bonds is inherently neglected in the aforementioned discussions. Only in situ physical characterization can validate or dismiss different models, but computational modeling can assist in the interpretation of experimental results.

The effects of substituents are twofold: (i) to lift the degeneracy of the bicyclic structure and (ii) to induce features specifically related to the attached functional group. We analyze these effects and compare different methyl isomers to see if surface spectroscopies can be used to enlighten proposed connections between adsorption sites and isomeric selectivity [11]. The 1- and 5-isomers represent different paths for the hydrogenation of 1-methylnaphthalene (1-MeNapht), and formation of neither isomer requires methyl migration. Identification of bicyclic ring structures is also of interest since these compounds find use as solvents and potential hydrogen sources for coal derived liquids.

\section{Materials and methods}

\subsection{Experimental}

The catalyst, $\mathrm{NiMo} / \mathrm{TiO}_{2}-\mathrm{Al}_{2} \mathrm{O}_{3}$, was sulfided (10 wt. $\% \mathrm{H}_{2} \mathrm{~S} / \mathrm{H}_{2}, 400{ }^{\circ} \mathrm{C}, 2 \mathrm{~h}, 1 \mathrm{~atm}$ ) prior to use [6]. Comparisons were also made with the same catalyst, $\mathrm{NiMo} / \mathrm{Al}_{2} \mathrm{O}_{3}-\mathrm{TiO}_{2}$, without presulfidation and with the empty matrix, $\mathrm{Al}_{2} \mathrm{O}_{3}-\mathrm{TiO}_{2}$. The feed, 1-MeNapht, was used as received (Aldrich, 98\%).

Microreactor studies addressed the temperature dependence of 1-MeNapht hydrogenation. A catalyst sample was repeatedly purged, and then exposed to the feed at room temperature, under 2 atm of hydrogen. The temperature was gradually increased, and the progress of the reaction followed by in situ diffuse reflectance infrared spectroscopy (Bomem Michelson MB 152 with a DTGS detector, $4 \mathrm{~cm}^{-1}$ ). The reactor was equipped with $\mathrm{NaCl}$ windows.

\subsection{Calculations}

The present study addressed the geometric and electronic structures of five bicyclic molecules: naphthalene, dilin, tetralin, octalin and decalin, their methyl-substituted analogues: 1-methylnaphthalene (1-MeNapht), 1-methyl-1,4-dihydronaphthalene (1-MeDilin), 1-methyl-1,2,3,4-tetrahydronahthalene (1-MeTetralin), 1-methyl-2,3,4,5,6,7,8-octahydronaphthalene (1-MeOctalin) and 1-methyl-decahydronaphthalene (1-MeDecalin), and two isomers: 5-methyl1,4-dihydronaphthalene (5-MeDilin) and 5-methyl1,2,3,4-tetrahydronaphthalene (5-MeTetralin). The decalins are synonymous with bicyclo[4,4,0] decane and 1-methyl bicyclo[4,4,0] decane. 
The electronic structures were evaluated with a Hartree-Fock Hamiltonian and 6-311G basis functions [12]. The molecular geometries represent the outputs from a Berny optimization algorithm. We compared the results from the HF/6-311G calculations with calculations using other basis sets and also a local exchange and correlation potential. Naphthalene has been evaluated in many different computational schemes, basis sets and Hamiltonians and our results are well in line with more elaborate schemes [13-16]. We also compared our results with published crystallographic data. Ionization potentials are preferably treated with a local exchange and correlation potential and a statistical approach to the excited state, but we have previously found that 'one-electron' energies of the excited states merely parallel the ground state orbital eigenvalues [17]. The main problem comes with vibrational frequencies where the Hartree-Fock approach overestimates the energies due to neglect of the anharmonicity of interatomic potentials. Expanded basis sets improve the agreement with experimental data, but computational work often reside to scaling.

We present total energies and one-electron ground state eigenvalues as they appear, with no fitting parameters. The densities of states (DoS) represent the Hartree-Fock ground state. This means that a rigid shift may have to be applied to all orbitals, when comparing our calculated DoS with experimental photoemission data. We artificially broaden each energy level with a Gaussian curve (1 eV FWHM) and summarize over all occupied orbitals to mimic the appearance of ultraviolet photoemission (UPS) spectra. The intensities are taken to be equal for all orbitals, i.e. we neglect the influence of excitation probabilities or matrix elements. We use the solid state term DoS since this is the common vocabulary in photoemisson studies of adsorbed species.

Vibrational frequencies were evaluated from the second derivatives at the stationary point of each converged geometry. Each absorption line was broadened by a $4 \mathrm{~cm}^{-1}$ (FWHM) Gaussian envelope. The intensities, i.e. the peak heights of each Gaussian curve, represent the dynamical dipole moments for each vibrational mode. These intensities can be modified by adsorption bond formation, as well as by the electrodynamic response of a metal catalyst. All vibrational frequencies are multiplied by 0.89 , chosen as a best fit scaling factor [18]. We note a good agreement between our data and observed infrared intensities for Napht and MeDecalin [19-21], albeit the calculated frequencies before scaling are too high $[14,16]$.

\section{Results and discussion}

\subsection{Hydrogenation products}

Fig. 1 shows the converged geometries of unsubstituted bicyclic structures. These geometries agree with experimental data and the results of more

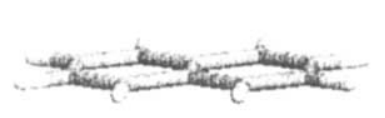

naphthalene

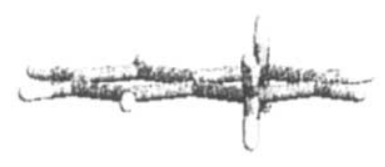

dilin

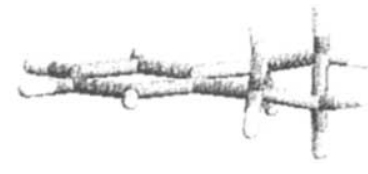

tetralin

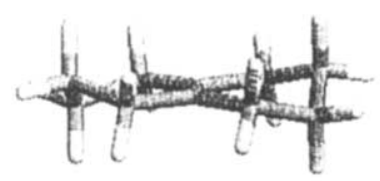

octalin

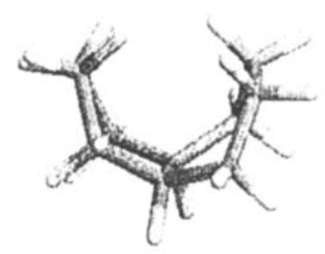

decalin

Fig. 1. Molecular graphics representation of the converged geometries of naphthalene, dilin, tetralin, octalin and decalin. The calculations represent the boat or cis-forms, where appropriate. 

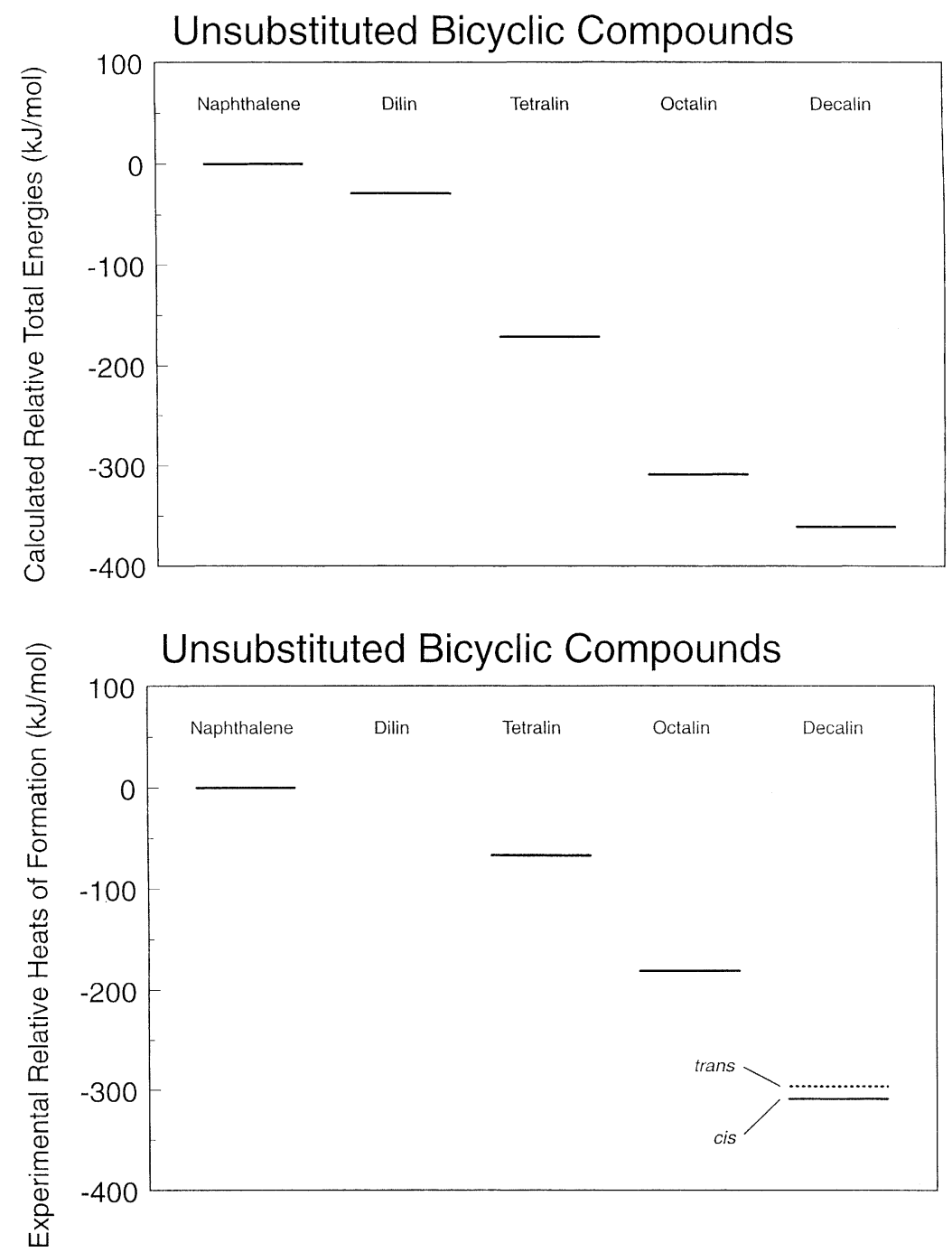

Fig. 2. Calculated total energies (a) and experimental heats of formation (b) for the hydrogenation of naphthalene to dilin, tetralin, octalin and decalin. The calculations represent the boat or cis-form of decalin.

elaborate computational schemes [22]. Decalin is represented by the more favorable boat or cis-form. It is only this species which can pass readily through the channels of zeolite-X [9]. Pd/zeolite-X is an effective catalyst for hydrocracking of the fully hydrogenated two-ring [7], but zeolitic catalysts may achieve high activity for hydrogenation as well as cracking, i.e. a direct one-step process from aromatic feeds to fuel components [8], if supported metal sulfides are added [23].
Fig. 2a presents calculated total energies for dilin, tetralin, octalin and decalin, with naphthalene and molecular hydrogen as reference. The calculated data are compared with standard heats of formation (Fig. 2b) [24]. Experimental and theoretical data show that complete hydrogenation can be favorable, but free energy evaluations and empirical knowledge give that aromatic products dominate at high temperature or limited hydrogen activity [10]. 


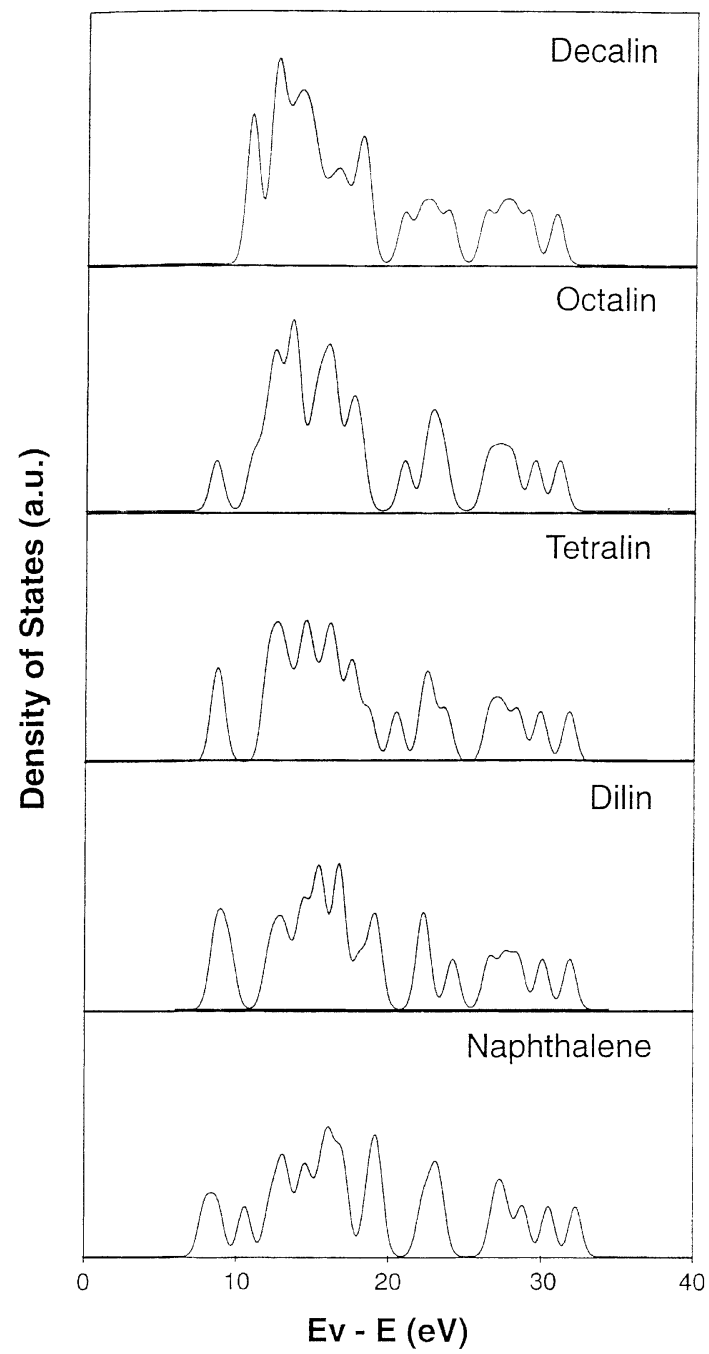

Fig. 3. Densities of states for naphthalene, dilin, tetralin, octalin and decalin. These data are to be compared with experimental valence band photoemission spectra. Note that the energies are given relative to the vacuum level.

\subsection{Identification of intermediates}

Fig. 3 shows DoS for the un-substituted compounds. The overall impression is that these DoS do not represent the distinct fingerprints necessary for in situ identification with UPS. The only striking difference is the reduced DoS at the highest energies, following hydrogenation. The density above $10 \mathrm{eV}$ is essentially non-bonding with respect to attached functional groups, until hydrogenated. Hydrogenation forms $\mathrm{C}-\mathrm{H} \sigma$-bonds, thereby shifting this density to lower energies. This brings similarities to free electron systems, since the $\pi$-electrons of large aromatic groups or chelates form the analogue of a free electron gas [25].

Unsaturated compounds react with hydrogen on metal or metal sulfide catalysts. These catalysts also have high DoS at the highest occupied orbitals, the Fermi level in solid state terminology. This means that orbitals at high energies, fingerprints of unsaturated compounds, will overlap with the catalyst states. This will make it difficult to discern and identify the adsorbates. One possibility is to use multielectron features in core level (ESCA) photoemission spectra.

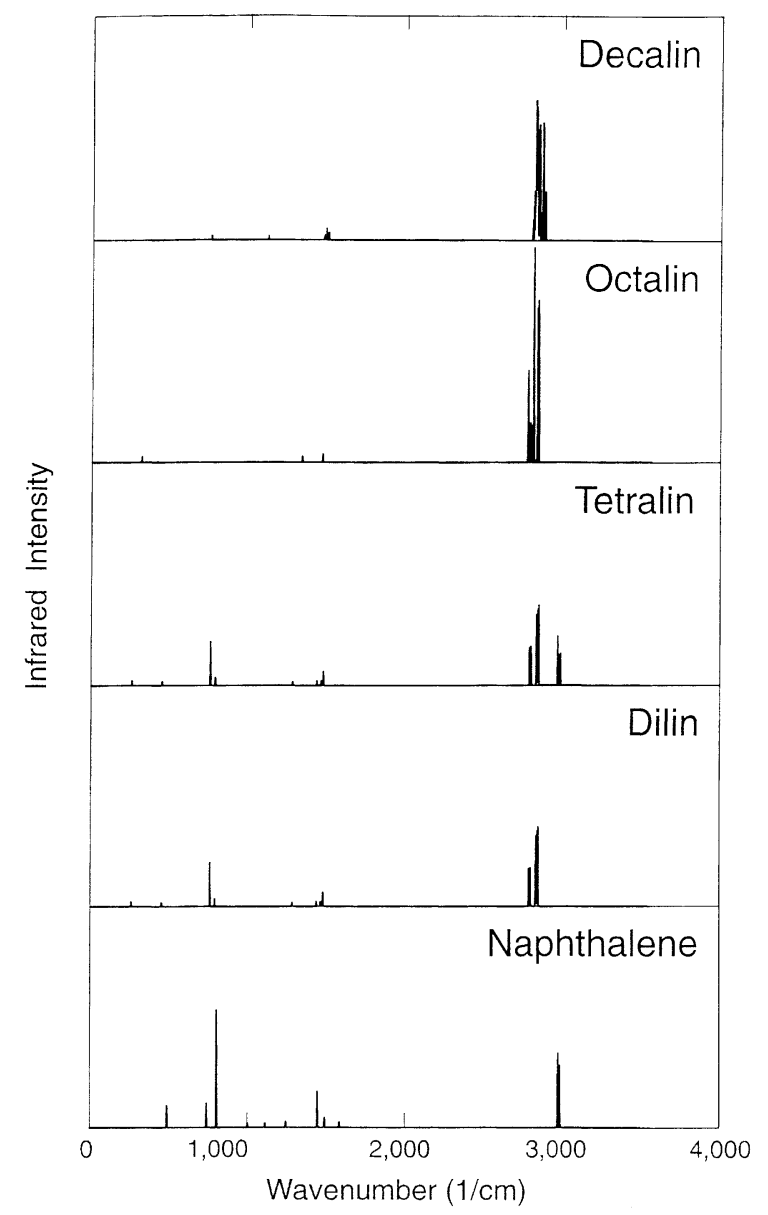

Fig. 4. Calculated vibrational (dipole) spectra for naphthalene, dilin, tetralin, octalin and decalin. 
Naphthalene has a characteristic shake up spectrum [26]. Another possibility is to use two photon energies, with different relative cross-sections for adsorbate and metal d-states.

Fig. 4 shows that the vibrational spectra are distinctly different for the bicyclic structures. The naphthalene spectrum has a characteristic low frequency band, and Fig. 4 shows that hydrogenation shifts intensity away from this region. This is the most characteristic feature of any spectroscopy considered in this work.

\section{$\mathrm{Al}_{2} \mathrm{O}_{3}-\mathrm{TiO}_{2} / \mathrm{NiMo} / \mathrm{preH}_{2} \mathrm{~S} / \mathrm{H}_{2}+\mathrm{MeNapht}$}

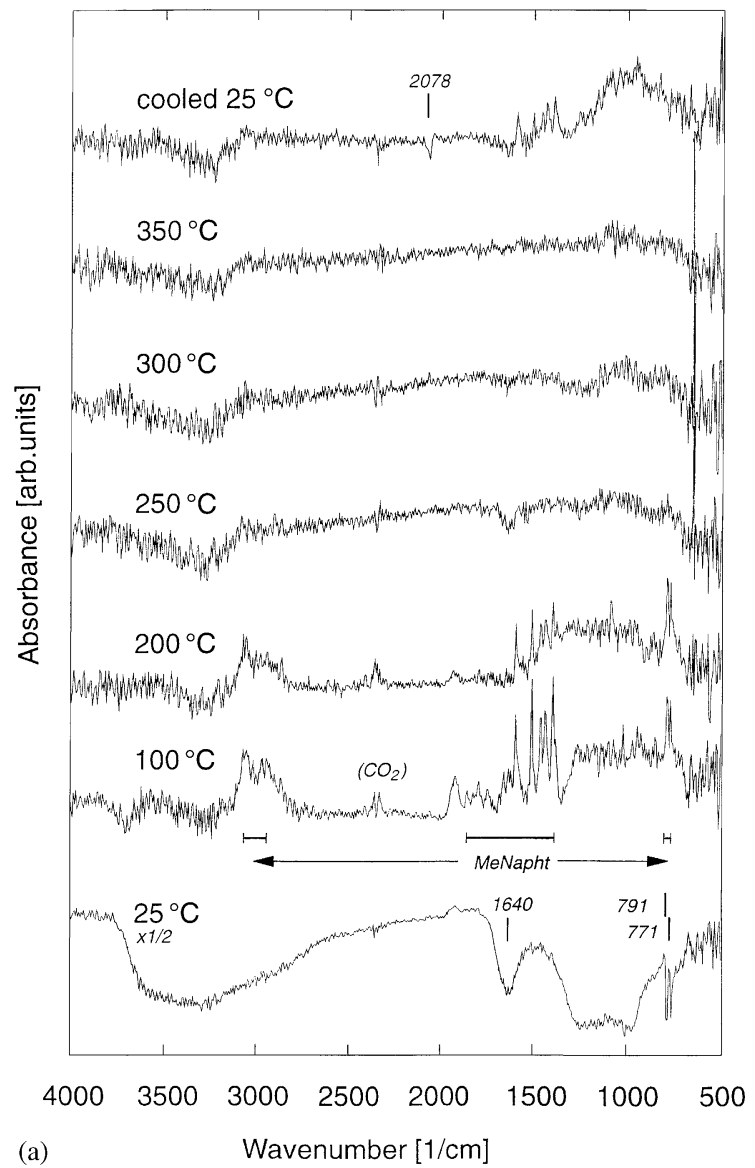

The experimental position for the low frequency naphthalene band is $780 \mathrm{~cm}^{-1}[19,21]$, compared with a calculated value of $803 \mathrm{~cm}^{-1}$ (Fig. 4). More elaborate calculations have been shown to reproduce the experimental energies even better [16]. Explicit calculations of hydrogenation and dehydrogenation reactions are non-trivial. One example shows that 1,4,5,8-tetrahydronaphthalene (isotetralin) is much more reactive than tetralin, due to the lower barrier for formation of 1,4-dihydronaphthalene [27], another example that free radicals may be important [28], but

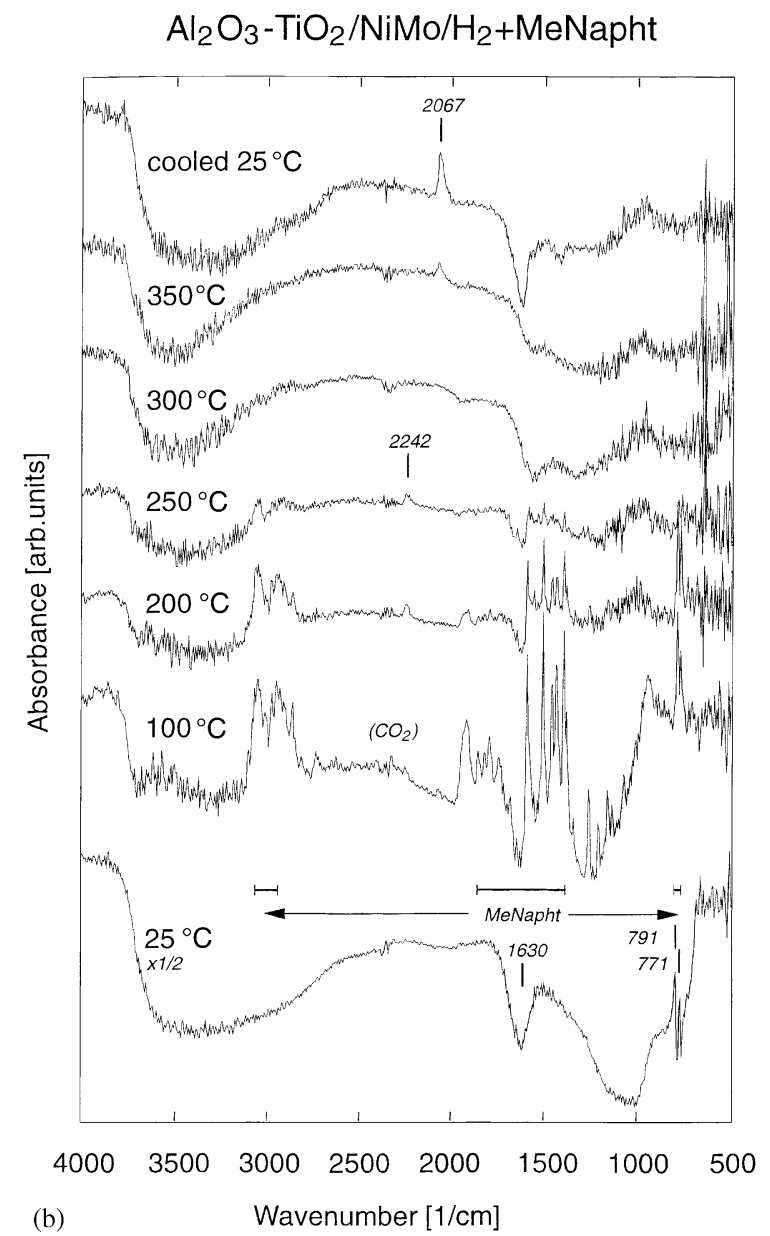

Fig. 5. In situ diffuse reflectance infrared spectra of 1-MeNapht adsorbed on: (a) presulfided $\mathrm{Al}_{2} \mathrm{O}_{3}-\mathrm{TiO}_{2} / \mathrm{NiMo}$, (b) $\mathrm{Al}_{2} \mathrm{O}_{3}-\mathrm{TiO}{ }_{2} / \mathrm{NiMo}$ without presulfidation and (c) $\mathrm{Al}_{2} \mathrm{O}_{3}-\mathrm{TiO}_{2}$. Each catalyst was purged with hydrogen at room temperature, pressurized at $p\left(\mathrm{H}_{2}\right)=2$ bar and saturated with MeNapht at $25^{\circ} \mathrm{C}$ (bottom spectra). The temperature was gradually increased. The topmost spectrum in each figure represents the catalyst after annealing to $400{ }^{\circ} \mathrm{C}$ (from $350{ }^{\circ} \mathrm{C}$ ) and recooling to $25^{\circ} \mathrm{C}$. Presulfided $\mathrm{Al}_{2} \mathrm{O}_{3}-\mathrm{TiO}_{2} / \mathrm{NiMo}$, annealed in pure hydrogen, was used as reference for (a), $\mathrm{Al}_{2} \mathrm{O}_{3}-\mathrm{TiO}_{2} / \mathrm{NiMo}$ without presulfidation, annealed in pure hydrogen, was used as reference for (b), and $\mathrm{Al}_{2} \mathrm{O}_{3}-\mathrm{TiO}_{2}$, annealed in pure hydrogen, was used as reference for (c). 


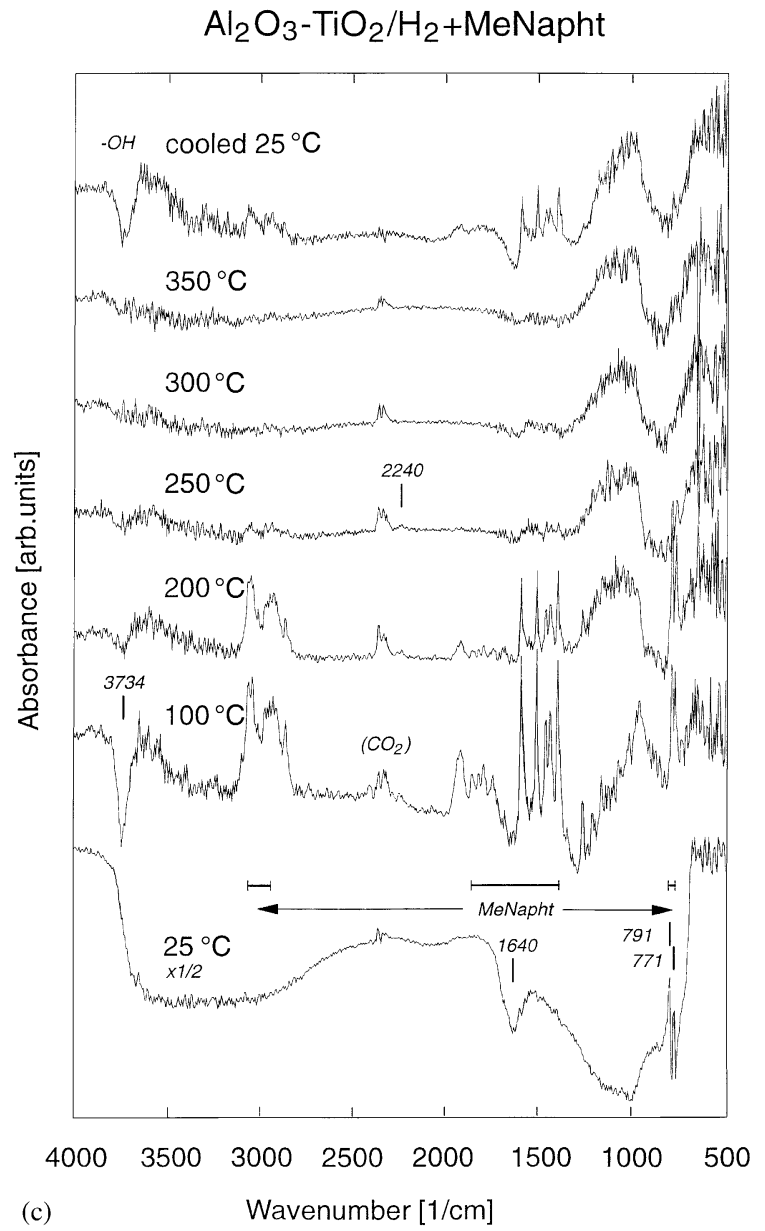

Fig. 5. (Continued).

neither of these studies take the adsorbed state into account.

\subsection{Surface species}

Fig. 5a-c shows in situ diffuse reflectance infrared spectra of 1-MeNapht, interacting with different catalysts, in a hydrogen atmosphere. Fig. 5a presents data for $\mathrm{Al}_{2} \mathrm{O}_{3}-\mathrm{TiO}_{2} / \mathrm{NiMo}$, reduced and presulfided in $\mathrm{H}_{2} / \mathrm{H}_{2} \mathrm{~S}$, Fig. $5 b$ data for $\mathrm{Al}_{2} \mathrm{O}_{3}-\mathrm{TiO}_{2} / \mathrm{NiMo}$, without sulfidation, and Fig. 5c interaction with the empty support, $\mathrm{Al}_{2} \mathrm{O}_{3}-\mathrm{TiO}_{2}$.

At $25^{\circ} \mathrm{C}$, the catalysts are soaked in MeNapht. No clear vibrational bands can be distinguished, except the low frequency double-peak at 771 and $791 \mathrm{~cm}^{-1}$.

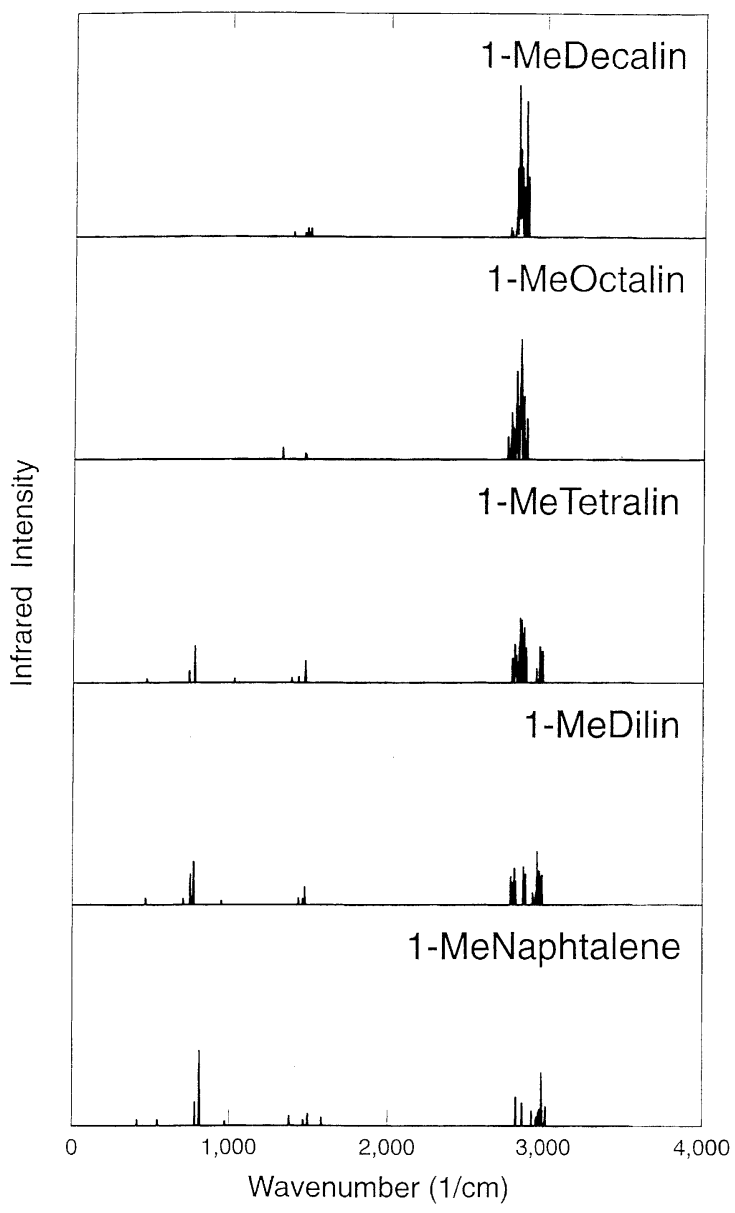

Fig. 6. Calculated vibrational (dipole) spectra for 1-MeNapht, 1-MeDilin, 1-MeTetralin, 1-MeOctalin and 1-MeDecalin.

The thick film distorts the peak shapes, but this double band is clearly identifiable in theoretically derived vibrational spectra (Fig. 6). The structure remains for MeDilin and MeTetralin, but fades away for MeOctalin and MeDecalin. The broad, anomalous peak at $1640 \mathrm{~cm}^{-1}$ (Fig. $5 \mathrm{a}-\mathrm{c}, 25^{\circ} \mathrm{C}$ ) comes from bulk MeNapht.

Hydrogenation shifts intensity from the low frequency region to the $\mathrm{C}-\mathrm{H}$ region, as was observed for the un-substituted two-rings (Fig. 4), but each vibrational band is split (Fig. 6). This split will be seen as a broadening of the bands, and comes because the methyl group lifts the degeneracy of internal vibrational modes, in each bicyclic structure. The explicit 
bands from the methyl ligand are comparatively weak and buried under these broadened envelops.

Intensity around $1500 \mathrm{~cm}^{-1}$ is present throughout the hydrogenation stages (Fig. 6). This makes this region less useful as a monitor, since it is not trivial to distinguish changes caused by hydrogenation from modifications caused by adsorption. We do observe a fingerprint of MeNapht in the experimental data (Fig. 5a-c), but no vibrational shifts are observed as a function of temperature, only a lowered intensity from 100 to $250^{\circ} \mathrm{C}$, which means that we observe the desorption of a largely unmodified species. The same features also reappear, due to readsorption, after cooling to $25^{\circ} \mathrm{C}$ (Fig. 5a-c, topmost spectra).

Recooling to $25^{\circ} \mathrm{C}$ results in recondensation of MeNapht (Fig. 5a and c), but also in a new feature at $2067 \mathrm{~cm}^{-1}$ (Fig. 5b). This has been assigned to $\mathrm{Ni}(\mathrm{CO})_{2}$ [29]. A second feature at $2242 \mathrm{~cm}^{-1}$ likely corresponds to a different partial oxidation product, possibly chemisorbed $\mathrm{CO}_{2}$ (Fig. $5 \mathrm{~b}$ and c).

Finally, we note the removal of intensity in the hydroxyl band region caused by MeNapht adsorption on the empty support $\mathrm{Al}_{2} \mathrm{O}_{3}-\mathrm{TiO}_{2}$ (Fig. 5c). The band position, $3734 \mathrm{~cm}^{-1}$, agrees with sites on the unmodified alumina, rather than acid sites, which appear around $3570 \mathrm{~cm}^{-1}$ [30]. The temperature dependence is vastly altered by MeNapht, but we conclude that the effect is rather non-discriminatory and that adsorbed MeNapht suppresses all hydroxyl groups.

\subsection{Substituted two-ring structures}

Vibrational spectra show promise as an in situ tool of two-ring hydrogenation reactions and of ring substitution (Figs. 5 and 6), but the value of photoemission is less clear. The DoS, i.e. the orbital fingerprint changes in the same way for the methyl-substituted compounds (Fig. 7) as for the un-substituted two-rings (Fig. 3). This means a reduced DoS close to the highest occupied orbitals as more double-bond character is removed. When trying to deduce the effect of the methyl group on the molecular orbitals, one notes minor changes for all substituted forms with respect to their un-substituted counterparts, but these changes are too vague to form the basis for identification with photoemission spectroscopy. Hence, it is not trivial to distinguish methylnaphthalene from naphthalene with valence band photoemission spectroscopy. The methyl

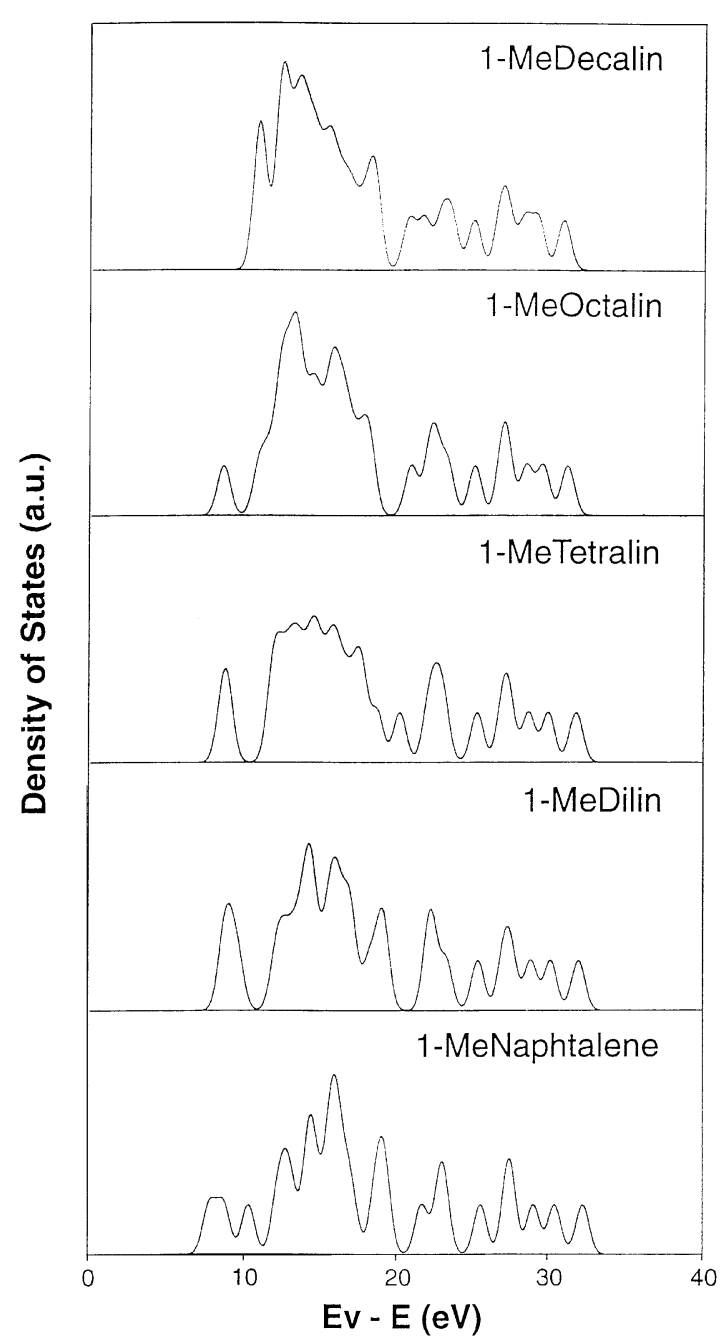

Fig. 7. Densities of states for 1-MeNapht, 1-MeDilin, 1-MeTetralin, 1-MeOctalin and 1-MeDecalin. These data are to be compared with experimental valence band photoemission spectra. Note that the energies are given relative to the vacuum level.

group, a common functional group, with a clear fingerprint when absorbed as an entity directly on the catalyst, gives an irrelevant signal compared with the bicyclic structure.

Figs. 8 and 9 show the geometries and energies of methyl-substituted two-ring structures. The geometries are similar to the structures of un-substituted two-rings (Fig. 1), but for a few subtle differences. It appears that the presence of a methyl group can influence the buckling for some partially hydrogenated 


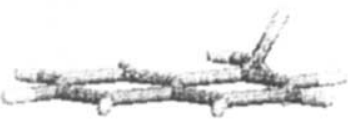

1-MeNapht

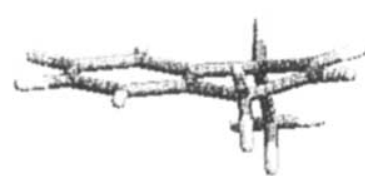

1-MeDilin

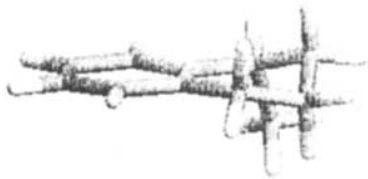

1-MeTetralin

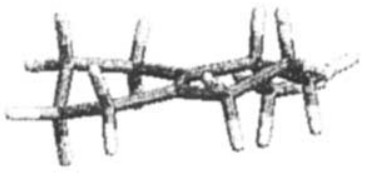

1-MeOctalin

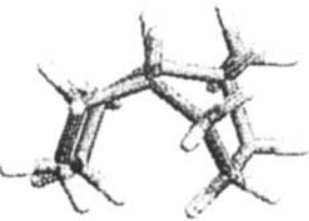

1-MeDecalin

Fig. 8. Molecular graphics representation of the converged geometries of 1-MeNapht, 1-MeDilin, 1-MeTetralin, 1-MeOctalin and 1-MeDecalin.

two-ring molecules. The energetics parallel the data of the un-substituted compounds (Fig. 2). Again we underline that explicit calculations of activation barriers are beyond the scope of the present work, but available data do not indicate that the methyl group is a center for bond activation, and the barrier should thus be similar as for the un-substituted two-rings.
Experimental data show that methyl groups, at large, remain at the same positions throughout the hydrogenation process $[6,31]$. This low migration activity suggests that $\mathrm{C}-\mathrm{C}$ bond activation, a necessary prerequisite for hydrogenation, takes place at the ring and that naphthalene and methylnaphthalene should display comparative barriers for hydrogenation.

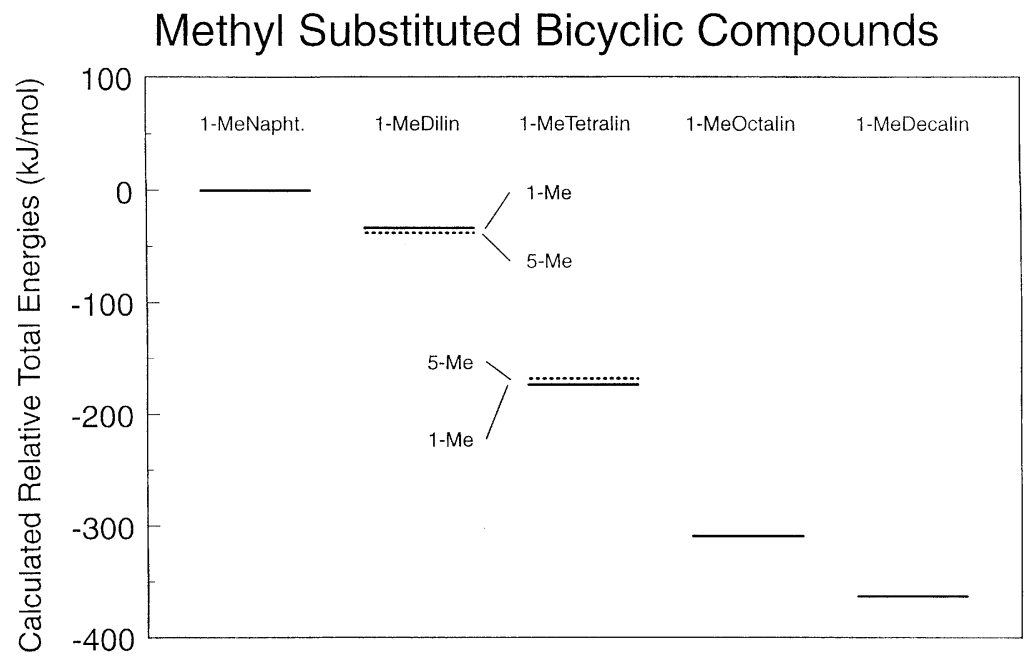

Fig. 9. Calculated total energies for the hydrogenation of 1-MeNapht to 1-MeDilin, 1-MeTetralin, 1-MeOctalin and 1-MeDecalin. The energies for the 5-MeDilin and 5-MeTetralin isomers are given as dashed lines. 

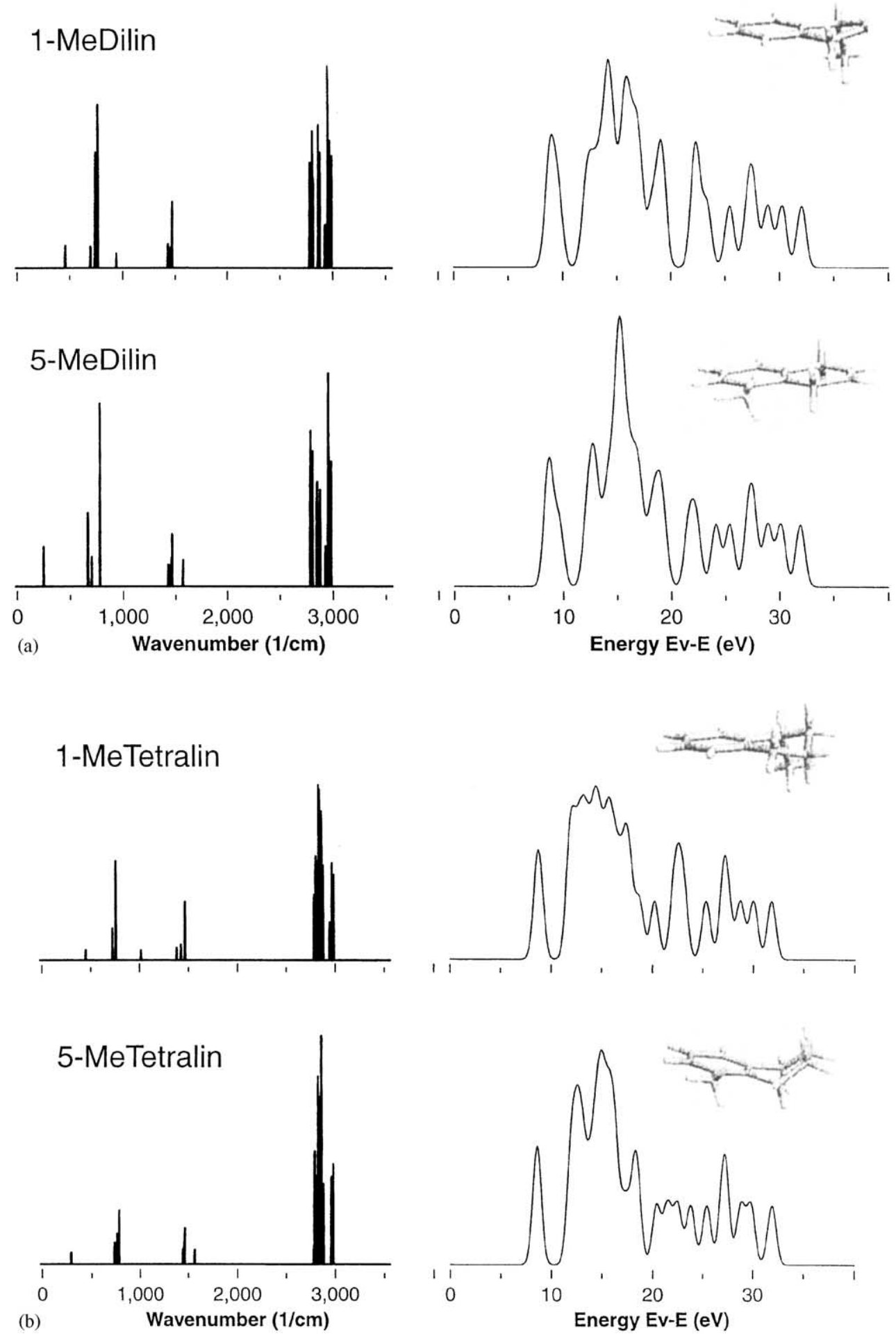

Fig. 10. Vibrational spectra and densities of states for: (a) 1-MeDilin and 5-MeDilin and (b) 1-MeTetralin and 5-MeTetralin. Converged geometries are also shown. 


\subsection{Isomers}

Isomeric selectivity for hydrogenation of substituted naphthalene has been related to specific adsorption sites for each position of the methyl group [11]. Correlations between specific site geometries and isomer distribution is a statistical measure to validate these ideas, but it is only through in situ techniques that we can observe intermediates.

Partial hydrogenation of 1-methylnaphthalene to methyldilin or methyltetralin can, without methyl migration, result in the attached functional group at either position 1 or at position 5. Position 5 corresponds to the methyl group bound to the remaining aromatic ring and position 1 to the methyl group bound to the fully (tetralin) or partly hydrogenated ring (dilin). Fig. 9 gave small energetic differences between different isomers, compared with the energy differences of hydrogenation. Fig. 10a and b also show the corresponding converged geometries. We have no experimental data for the yields of different isomers during partial hydrogenation, but a $10 \mathrm{~h}$ experiment at $325^{\circ} \mathrm{C}$ yielded more than $95 \%$ MeDecalin from 1-MeNapht [6]. A small fraction of the two-rings underwent isomerization reactions but eight out of nine molecules were characterized as $1-\mathrm{Me}$ substituted. The 1- and 5-methyl isomers are symmetry equivalent for decalin. This low yield of methyl migration motivated us to neglect other isomers.

Fig. 10a and $\mathrm{b}$ also show the expected valence band photoemission spectra and vibrational data of MeDilin and MeTetralin with the methyl group at positions 1 and 5. It is obvious that only subtle differences result from the different positions of the methyl ligand, differences easily overshadowed by modifications induced by interaction with the catalyst. It is therefore unlikely that either photoemission or vibrational spectra will have any bearing on models for isomeric selectivity at different adsorption sites.

Fig. 10a and $\mathrm{b}$ show that the planarity differs between the 1-methyl and 5-methyl isomers of 1,2,3,4tetrahydronaphthalene and 1,4-dihydronaphthalene and the un-substituted forms of tetralin and dilin (Fig. 1). Bulky substituents need space to accommodate the larger van der Waals radii. This means that methyl substitution on a hydrogenated carbon, which positions the functional group to one side of the ring, commonly bends the outer atoms away into a boat shape. Substitution on an aromatic carbon places the symmetry axis of the methyl ligand in the molecular plane, with no resulting extra incentive for non-planarity.

\section{Conclusions}

We observed adsorbed MeNapht, below $250{ }^{\circ} \mathrm{C}$, on presulfided $\mathrm{NiMo} / \mathrm{Al}_{2} \mathrm{O}_{3}-\mathrm{TiO}_{2}$ catalysts, $\mathrm{NiMo} / \mathrm{Al}_{2}$ $\mathrm{O}_{3}-\mathrm{TiO}_{2}$ catalysts and empty supports $\mathrm{Al}_{2} \mathrm{O}_{3}-\mathrm{TiO}_{2}$. MeNapht adsorption suppressed $\mathrm{OH}$ groups nondiscriminatory on the empty support and the metal loaded catalysts.

Theoretical modeling showed that vibrational spectroscopy is a sensitive tool for studies of naphthalene hydrogenation. The dynamic dipole moments of characteristic bands in the mid-IR regime were significant, which vouch for high sensitivity. Naphthalene, dilin, tetralin and octalin were readily distinguishable, but decalin and octalin showed similar patterns. Shifts induced by adsorbent-catalyst interactions will modify the fingerprints of free molecules, and we observed liquid MeNapht as well as an adsorbed species by in situ IR. Photoemission in the valence band region (UPS or XPS/ESCA) is less useful. Methylation causes no significant change of the DoS, but a characteristic broadening of all vibrational bands.

Conclusively mid-range infrared spectroscopy offers the possibility, which photoemission lacks, to follow surface reactions of bicyclic compounds in situ. Only subtle differences, between the 1- and 5-isomers of partially hydrogenated 1-methylnaphthalene, makes the prospect to study methyl migration and isomerization reactions less promising.

Our total energies data agreed with experimental findings and pointed at decalins, methyl- and un-substituted, as the dominant products, with tetralins as abundant intermediates, and dilins and octalins as short lived transient stages.

\section{References}

[1] J.F. Patzer, R.J. Farrauto, A.A. Montagna, Ind. Eng. Chem. Proc. Des. Dev. 18 (1979) 625.

[2] M.J. Girgis, B.C. Gates, Ind. Eng. Chem. Res. 30 (1991) 2021.

[3] H. Topsoe, B.S. Clausen, F.E. Massoth, Hydrotreatment Catalysis, Springer, Berlin, 1996. 
[4] Th. Weber, R. Prins, R.A. van Santen (Eds.), Transition Metal Sulphides, Chemistry and Catalysis, Kluwer Academic Publishers, Dordrecht, 1998.

[5] A. Stanislaus, B.H. Cooper, Catal. Rev. Sci. Eng. 36 (1994) 75.

[6] B. Demirel, W.H. Wiser, Fuel Process. Technol. 53 (1997) 157.

[7] B. Demirel, W.H. Wiser, A.G. Oblad, W. Zmierczak, J. Shabtai, Fuel 77 (1998) 301.

[8] B. Demirel, PhD Thesis, University of Utah, Salt Lake City, 1996.

[9] Ş. Sayan, B. Demirel, J. Paul, Fuel 79 (2000) 1395.

[10] B. Demirel, W.H. Wiser, Fuel Process. Technol. 55 (1998) 83.

[11] J. Shabtai, N.K. Nag, F.E. Massoth, in: Proceedings of the 9th International Congress on Catalysis, 1988, p. 1.

[12] M. Frisch, Æ. Frisch, J.B. Foresman, Gaussian 94, User's Reference, Gaussian Inc., Pittsburgh, 1995.

[13] G.B. Bacskay, N.S. Hush, S.J. Ikuta, J. Phys. Chem. 95 (1991) 9291.

[14] E. Cebe, G. Grampp, Z. Phys. Chem. 187 (1994) 15.

[15] C. Niederalt, S. Grimme, S.D. Peyerimhoff, Chem. Phys. Lett. 245 (1995) 455.

[16] S.R. Langhoff, J. Phys. Chem. 100 (1996) 2819.

[17] Y. Shao, J. Paul, O. Axelsson, F.M. Hoffmann, J. Phys. Chem. 97 (1993) 7652.
[18] J.B. Foresman, Æ. Frisch, Exploring Chemistry with Electronic Structure Methods, Gaussian Inc., Pittsburgh, 1996.

[19] R.J. Keller (Ed.), The Sigma Library of FT-IR Spectra, 1st Edition, Sigma, St. Louis, 1986.

[20] C.J. Pouchert (Ed.), The Aldrich Library of FT-IR Spectra, Vapor Phase, Vol. 3, Aldrich, Milwaukee, 1989.

[21] D.R. Lide, G.W.A. Milne (Eds.), Handbook of Data on Common Organic Compounds, CRC Press, Boca Raton, 1995.

[22] J.E. Bloor, M. Eckert-Maksič, M. Hodošček, Z.B. Maksič, K. Poljanec, N. J. Chem. 17 (1993) 157.

[23] Y. Okamoto, H. Katsuyama, Ceram. Process. 43 (1997) 2809.

[24] CRC Handbook of Chemistry and Physics, 65th Edition, Robert C. Weast (editor-in-chief) CRC Press, Cleveland, 1984.

[25] J. Paul, M.L. Smith, K.G. Paul, Biochim. Biophys. Acta 832 (1985) 257.

[26] C. Enkvist, S. Lunell, B. Sjögren, P.A. Bruehwiler, S. Svensson, J. Chem. Phys. 103 (1995) 6333.

[27] M.W. Bedell, C.W. Curtis, Prep. Pap. Am. Chem. Soc. Div. Fuel Chem. 36 (1991) 425.

[28] H.A. Kurtz, R.V. Lloyd, R.V. Williams, J. Org. Chem. 52 (1987) 302.

[29] NIST Chemistry WebBook, http://webbook.nist.gov/chemistry.

[30] L.O. Öhman, J. Paul, Mater. Chem. Phys. 73 (2002) 242.

[31] B. Demirel, W.H. Wiser, in: Proceedings of the 212th ACS National Meeting, Fuel Chem. 41 (1996) 1099 (preprint). 